\title{
VLA and BIMA observations toward the exciting source of the massive HH 80-81 outflow
}

\author{
Y. Gómez, L.F. Rodríguez \\ Centro de Radioastronomía y Astrofísica, UNAM, Apdo. Postal 3-72 (Xangari) 58089 \\ Morelia, Michoacán, México \\ y.gomez@astrosmo. unam.mx; 1.rodriguez@astrosmo.unam.mx \\ J. M. Girart \\ Departament d'Astronomia i Meteorologia, Universitat de Barcelona, Av. Diagonal 647, \\ 08028 Barcelona, Catalunya, Spain \\ jgirart@ub.edu \\ G. Garay \\ Departamento de Astronomía, Universidad de Chile, Casilla 36-D, Santiago, Chile \\ guido@das.uchile.cl \\ and \\ J. Martí \\ Departamento de Física, Escuela Politécnica Superior, Universidad de Jaén, Calle Virgen \\ de la Cabeza, 2, E-23071 Jaén, Spain \\ jmarti@ujaen.es
}

\begin{abstract}
We present high angular resolution VLA and BIMA observations of $\mathrm{NH}_{3}$, $\mathrm{HCO}^{+}, \mathrm{HCN}$ and $\mathrm{SO}$ molecular emission and 1.4, 3.5 and $7 \mathrm{~mm}$ continuum emission toward the exciting source of the HH 80-81 system. This object is one of the few massive protostars known to be driving a collimated outflow. We report the first detection of SO $5_{5}-4_{4}$ molecular emission toward the exciting source of $\mathrm{HH}$ 80-81, suggesting that this transition may be a good tracer of molecular gas near massive protostars. We also detected toward this source dust continuum emission at 1.4 and $3.5 \mathrm{~mm}$. From the $\mathrm{SO}$ molecular emission and the dust emission we roughly estimated that the molecular mass associated with the circumstellar surroundings of the exciting source of the thermal jet is in the range $1-3 \mathrm{M}_{\odot}$. Weak and broad $(2,2)$ ammonia emission was also found in the
\end{abstract}


direction of the jet suggesting the presence of small amounts of molecular gas at high temperatures $(>50 \mathrm{~K})$.

The VLA observations show the presence of three ammonia components toward the $\mathrm{HH} 80-81$ region. The brightest component peaks at $\sim 8^{\prime \prime}$ to the $\mathrm{NE}$ of the thermal jet and is associated with the $\mathrm{H}_{2} \mathrm{O}$ maser spots in the region. A second ammonia clump is located about $25^{\prime \prime}$ to the NE of the jet and is associated with class I methanol masers. The third ammonia component is located $1^{\prime}$ to the south from the thermal jet and may be a molecular core yet without stellar formation. The BIMA observations show that the strongest emission in the $\mathrm{HCO}^{+}$and $\mathrm{HCN}$ lines originate close to the $\mathrm{H}_{2} \mathrm{O}$ maser, and cover the same spatial region and velocity range as the brightest ammonia component.

Subject headings: ISM: outflows-individual(HH 80-81, GGD 27, IRAS 181622048) — ISM: HII regions - molecules — radio lines - ISM: Herbig-Haro objects astrochemistry

\section{Introduction}

The HH 80-81 system, also known as GGD 27 (Gyulbudaghian et al. 1978), consists of a highly collimated radio jet that powers an outflow with a total projected extent of 5.3 pc, at an adopted distance of 1.7 kpc (Rodríguez et al. 1980; Martí, Rodríguez, \& Reipurth $1993 ; 1995 ; 1998)$. The thermal radio jet associated with HH 80-81 terminates to the south in the extremely bright optical HH objects 80 and 81 (Reipurth \& Graham 1988; Martí, Rodríguez, \& Reipurth 1993, 1995; Heathcote, Reipurth, \& Raga 1998) and to the north in the radio source HH 80 North (Martí et al. 1993; Girart et al. 1994; 2001). The central radio continuum source has a bright far-infrared counterpart, IRAS 18162-2048, which implies the presence of a luminous $\left(\sim 2 \times 10^{4} \mathrm{~L}_{\odot}\right)$ young star or cluster of stars (Aspin \& Geballe 1992). Water maser emission has been reported in the vicinity of the central radio continuum source (Rodríguez et al. 1978; 1980). The $\mathrm{H}_{2} \mathrm{O}$ masers do not coincide with the jet core, suggesting that they are being powered by a different star (Gómez, Rodríguez, \& Martí 1995; Martí, Rodríguez, \& Torrelles 1999). Methanol emission at $95 \mathrm{GHz}$ has been reported by Val'tts et al. (2000) to the northeast of the exciting source of the jet at the position of $\alpha(2000)=18^{h} 19^{m} 12.89 ; \delta(2000)=-20^{\circ} 47^{\prime} 13^{\prime \prime}$. 6 with a positional error of $10^{\prime \prime}$. These methanol masers are classified as class I which are believed to trace high-mass protostars that have not significantly ionized their environment.

The most remarkable characteristic of the $\mathrm{HH} 80-81$ system is the high degree of collimation of the jet, with an opening angle of $\sim 1^{\circ}$ (Martí, Rodríguez, \& Reipurth 1993). 
Collimated outflows and accretion disks are thought to be present at the same evolutionary stage in the formation of both low-mass and high-mass stars (e.g. Garay \& Lizano 1999; Kurtz 2000). An accretion disk around the central star is believed to be responsible for the ejection and collimation of the gas. In contrast with low-mass stars, where disk structures have been observed commonly around young objects (Beckwith \& Sargent 1996; Wilner, Ho, \& Rodríguez 1996; Rodríguez, et al. 1998; Wilner et al. 2000), in high-mass stars it has been difficult to establish the presence of accretion disks. Whether the massive protostars are formed by accretion (as it is the case for low mass stars) or by merging of protostellar condensations (Bonnell \& Bate 2002) remains controversial. It has been argued, for example, that the ionizing flux present in massive stars could rapidly disrupt the disk. However, the presence of well collimated outflows in a few objects also points to the presence of accretion disks surrounding massive stars. If massive OB stars are formed by accretion, we expect that disks and jets will be present in their earliest stages of evolution, as in the case of low mass stars. Accretion disks and collimated outflows around B-type protostars have been tentatively reported toward Cepheus A HW2 (Rodríguez et al. 1994; Torrelles et al. 1996), IRAS 20126+4104 (Cesaroni et al. 1997; Zhang, Hunter \& Sridharan 1998), G192.16-3.82 (Shepherd et al. 1998; Devine et al. 1999; Shepherd, Claussen, \& Kurtz 2001), AFGL 5142 (Zhang et al. 2002) and AFGL 2591 (Trinidad et al. 2003). A possible collimated outflow associated with a luminous O-type protostar is IRAS 16547-4247, a bright infrared source that is associated with a triple radio source consisting of a compact central object and two outer lobes (Garay et al. 2003). There is, however, no known unquestionable case of a circumstellar disk associated with a massive O-type protostar.

The luminosity of $\mathrm{HH} 80-81$ is $\sim 2 \times 10^{4} \mathrm{~L}_{\odot}$, equivalent to that of a B0 ZAMS star. Since HH 80-81 possesses an extraordinary well collimated jet, it seems reasonable to propose that it should be originating from an accretion disk. However, until now there has been no clear evidence for the existence of this disk. A bipolar CO outflow has been detected and mapped in the direction of $\mathrm{HH} 80-81$ by Yamashita et al. (1989) which spatially coincides with the infrared reflection nebula in the region. Dense molecular gas traced by $\mathrm{NH}_{3}(1,1)$ emission was reported by Torrelles et al. (1986) from single dish observations, whereas Girart et al. (1994) reported the detection of $(1,1)$ and $(2,2)$ inversion transitions of ammonia downstream in HH 80 North. Also a CS molecular clump has been observed towards the exciting source of $\mathrm{HH}$ 80-81 which exhibits an elongated structure $\left(\sim 60 \times 25^{\prime \prime}\right)$ perpendicular to the CO molecular outflow, that has been interpreted as a molecular disk (Yamashita et al. 1989). However, high angular resolution observations are needed in order to establish the existence of a more compact circumstellar disk. We consider $\mathrm{HH} \mathrm{80-81} \mathrm{as} \mathrm{one} \mathrm{of} \mathrm{the} \mathrm{best} \mathrm{candidates}$ for the detection of a protoplanetary disk using molecular lines. Accordingly, the main goal of this work was to make observations, in several molecular lines and with adequate angular 
resolution, towards the core of $\mathrm{HH}$ 80-81 to search for an accretion disk that could account for the collimated outflow.

\section{Observations}

\subsection{VLA}

The $1.3 \mathrm{~cm}$ line and continuum observations were made on 1999 April 12 with the Very Large Array (VLA) of the $\mathrm{NRAO}^{1}$. The array was in the D configuration giving an angular resolution of $4^{\prime \prime}$ at $1.3 \mathrm{~cm}$. Structures larger than $60^{\prime \prime}$ were undetectable. We observed the $(\mathrm{J}, \mathrm{K})=(1,1)$ and $(2,2)$ inversion transitions of the ammonia molecule in the spectral line mode towards the core of the HH 80-81 complex. We assumed rest frequencies of 23694.496 and $23722.633 \mathrm{MHz}$ for the $(1,1)$ and $(2,2)$ transitions, respectively. We used a bandpass of $3.125 \mathrm{MHz}$, centered at an LSR velocity of $10 \mathrm{~km} \mathrm{~s}^{-1}$ and 63 spectral channels $48.8 \mathrm{kHz}$ wide each ( $\sim 0.6 \mathrm{~km} \mathrm{~s}^{-1}$ at the observing frequencies) plus a continuum channel containing the central $75 \%$ of the total band. Flux densities were bootstrapped from observations of $1331+305$, which was assumed to have a flux density of $2.4 \mathrm{Jy}$ at $1.3 \mathrm{~cm}$. The bandpass calibrator was $1229+020$ with a flux density of 28.6 Jy and the phase calibrator was 1733-130 with a flux density of $3.8 \mathrm{Jy}$ at $1.3 \mathrm{~cm}$. The data were edited and calibrated in the standard manner using the Astronomical Image Processing System (AIPS) developed by the NRAO.

All spectra were Hanning smoothed to a velocity resolution of $1.2 \mathrm{~km} \mathrm{~s}^{-1}$. The $r m s$ noise levels in a single spectral channel, after Hanning smoothing, were 2.5 and $2.2 \mathrm{mJy} \mathrm{beam}^{-1}$ (corresponding to 0.38 and $0.33 \mathrm{~K}$ ) for the $(1,1)$ and $(2,2)$ observations, respectively. The system temperature during observations was in the order of $150 \mathrm{~K}$. We applied a primary beam correction (with the task PBCOR of AIPS) to the ammonia data in order to have a good estimate of the flux for the emission that is displaced from the phase center.

\footnotetext{
${ }^{1}$ The National Radio Astronomy Observatory is operated by Associated Universities, Inc., under cooperative agreement with the National Science Foundation
} 


\subsection{BIMA}

The observations were carried out in 1999 September 27 at $3.5 \mathrm{~mm}$ and 2001 May 6 at $1.4 \mathrm{~mm}$ with the 10-antenna BIMA array ${ }^{2}$ in the $\mathrm{C}$ configuration. The phase calibrator used was 1733-130. Absolute flux calibration was performed by observing Mars. System temperatures were in the $200-400 \mathrm{~K}$ range at $3.5 \mathrm{~mm}$ and in the $1600-4000 \mathrm{~K}$ range at $1.4 \mathrm{~mm}$. The $3.5 \mathrm{~mm}$ frequency setup included the $\mathrm{HCO}^{+} 1-0$ and $\mathrm{HCN} 1-0$ lines, whereas the $1.4 \mathrm{~mm}$ setup included the $\mathrm{SO} 5_{5}-4_{4}$ and $\mathrm{H}_{2} \mathrm{CO} 3_{0,3}-2_{0,2}$ lines. Self-calibration was performed in the $1.4 \mathrm{~mm}$ data by using the continuum emission. Maps were made with the $(u, v)$ data weighted by the associated system temperatures. More details of the observations are given in Table 1.

\section{RESULTS}

\subsection{VLA and BIMA continuum emission}

Using the VLA we detected an unresolved $(\leq 2$ ".6 $1.3 \mathrm{~cm}$ continuum source coincident in position with the exciting source of the thermal jet. The estimated flux density at $1.3 \mathrm{~cm}$ is $6 \pm 1 \mathrm{mJy}$. This is consistent with the value reported by Martí, Rodríguez \& Torrelles (1999). The $7 \mathrm{~mm}$ flux density was obtained from data taken with the VLA on 1994 April 15. The unresolved $(\leq 0$ ". 4$) 7 \mathrm{~mm}$ source is shown in Figure 1 . The continuum images at 3.5 and $1.4 \mathrm{~mm}$ made with BIMA are shown on Figure 2. To discuss the nature of this emission we present in Figure 3 the continuum spectrum towards the exciting source of the thermal jet at $\mathrm{cm}$ and $\mathrm{mm}$ wavelengths. The 20,6, and $2 \mathrm{~cm}$ flux densities are taken from Martí et al. (1993), while the $1.3 \mathrm{~cm}$ data point is that presented above from our VLA observations. The flux densities at 7, 3.5 and $1.4 \mathrm{~mm}$ toward the exciting source of the outflow are listed in Table 2. From Figure 3 we can see that the $\mathrm{cm}$ emission shows the characteristic flat spectrum of nearly optically thin free-free emission $\left(\mathrm{S}_{\nu} \sim \nu^{0.2 \pm 0.1}\right)$, but that at $7 \mathrm{~mm}$ and shorter wavelengths, the flux density rises rapidly. This spectrum suggests that the flux densities at 3.5 and $1.4 \mathrm{~mm}$ are completely dominated by the dust (at $3.5 \mathrm{~mm}$ the expected contribution from the thermal jet is only $\sim 0.01 \mathrm{Jy}$ ). The spectral index derived from the $7,3.5$ and $1.4 \mathrm{~mm}$ fluxes is 2.6, consistent with optically-thin dust emission. There is also $1.4 \mathrm{~mm}$ emission associated with the $\mathrm{C}$ ammonia component (see Figure 2, and Table 3).

\footnotetext{
${ }^{2}$ The BIMA array is operated by the Berkeley-Illinois-Maryland Association with support from the National Science Foundation.
} 


\subsection{VLA spectral line emission}

The $(1,1)$ and $(2,2)$ main inversion transitions of ammonia were strongly detected in emission toward the northeast of the jet, but only very weak $(2,2)$ emission was observed at the position of the exciting source of the thermal jet.

Figure 4 shows the velocity-integrated ammonia $(1,1)$ emission toward $\mathrm{HH} 80-81$. We identify three main ammonia structures; the bright, $\mathrm{C}$ component which exhibits an extended structure and peaks close to the position of the water masers in the region, a small ammonia clump to the northeast of the $\mathrm{C}$ component (hereinafter called the NE component) and the $\mathrm{S}$ component which is located $\sim 1^{\prime}$ to the south of the $\mathrm{C}$ component.

Figure 5 shows the channel maps of the $\mathrm{NH}_{3}(1,1)$ and $(2,2)$ emissions toward the surroundings of the exciting source of $\mathrm{HH}$ 80-81. In general, the ammonia emission from the molecular cloud in both transitions appears distributed to the northeast of the thermal jet, spanning over the same velocity range, from $\sim 10$ to $14 \mathrm{~km} \mathrm{~s}^{-1}$. As noted before, the peak emission of the $\mathrm{C}$ ammonia component is nearly coincident with the position of the $\mathrm{H}_{2} \mathrm{O}$ maser spots (Gómez, Martí, \& Rodríguez 1995; Martí, Rodríguez \& Torrelles 1999).

Figure 6 presents a close-up of the velocity-integrated emission in the $(1,1)$ and $(2,2)$ ammonia lines from the $\mathrm{C}$ component and the $(1,1)$ line from the $\mathrm{S}$ component. The top panel also shows the $(1,1)$ ammonia emission from the NE ammonia clump where methanol maser emission has been reported (Val'tts et al. 2000). The S component was detected only in the $(1,1)$ ammonia transition and in the velocity range from 11.9 to $13.7 \mathrm{~km} \mathrm{~s}^{-1}$, suggesting it corresponds to a cold molecular clump.

The spectra of the $\mathrm{NH}_{3}(1,1)$ and $(2,2)$ inversion transitions from each individual ammonia component (integrated over their whole area of emission) are presented in Figure 7. The $(1,1)$ main and satellite lines were detected toward each individual ammonia component, while in the $(2,2)$ ammonia transition emission was detected only in the main line and toward the $\mathrm{C}$ and $\mathrm{NE}$ components. In Table 3 we list the ammonia parameters of the main and satellite ammonia lines from the $\mathrm{C}, \mathrm{NE}$ and $\mathrm{S}$ components determined by fitting a Gaussian profile to the corresponding spectrum. The peak intensity of these three ammonia components are around the LSR velocity of $+12 \mathrm{~km} \mathrm{~s}^{-1}$.

\subsection{BIMA spectral line emission}

Figure 8 shows line profiles and maps of the $\mathrm{SO}_{5} 5_{-}-4_{4}, \mathrm{HCO}^{+} 1-0$ and $\mathrm{HCN} 1-0$ emission. The SO emission arises from a compact region around the thermal jet. A Gaussian fit to 
the SO profile gives a peak flux density of $7.6 \pm 1.6 \mathrm{Jy}$, a central velocity of $\mathrm{V}_{L S R}=12.1$ $\pm 0.2 \mathrm{~km} \mathrm{~s}^{-1}$ and a linewidth of $\Delta \mathrm{V}=1.3 \pm 0.4 \mathrm{~km} \mathrm{~s}^{-1}$. This is the first time that $\mathrm{SO}$ emission is detected toward the exciting source of $\mathrm{HH}$ 80-81 and suggests that this transition may be a good tracer of molecular gas near massive protostars. The $\mathrm{HCO}^{+}$and $\mathrm{HCN}$ emissions trace basically the same spatial region as the ammonia central component and in a similar velocity range. Weak $\mathrm{HCO}^{+}$emission is observed toward the $\mathrm{NE}$ ammonia clump, confirming its existence. There is some enhancement of the $\mathrm{HCO}^{+}$and $\mathrm{HCN}$ emission toward the direction of the thermal radio jet, but it is difficult to separate the molecular gas arising from the jet region from the more extended central component. Despite the similitude of the $\mathrm{HCO}^{+}$and $\mathrm{HCN}$ integrated maps with the integrated ammonia maps, the observed $\mathrm{HCO}^{+}$ and HCN spectra show a clear self-absorption feature at the center of their lines (see Fig. 8), which is not observed in the ammonia lines. It is possibly a true self-absorption since the shortest baselines also show this absorption feature.

We also searched for emission in the $\mathrm{H}_{2} \mathrm{CO} 3_{0,3}-2_{0,2}$ line toward the region. The integrated velocity spectrum in a velocity range from 10 to $14 \mathrm{~km} \mathrm{~s}^{-1}$ did not show emission at a $3-\sigma$ level of $\sim 2 \mathrm{Jy}^{\text {beam }^{-1}}$.

\section{DISCUSSION}

The line data presented here reveal the presence of three main molecular structures that we called the $\mathrm{C}$, the $\mathrm{NE}$, and the $\mathrm{S}$ components. In what follows we discuss each of these components and derive their bulk physical parameters, and conclude with a discussion of the molecular and dust emission toward the surroundings of the jet.

\subsection{The $\mathrm{C}$ component}

This large structure of molecular gas has a peak position located about $8^{\prime \prime}$ northeast from the exciting source of the thermal jet. Emission toward this component was detected in the $(1,1)$ and $(2,2)$ transitions of $\mathrm{NH}_{3}$, and $\mathrm{HCN}(1-0)$ and $\mathrm{HCO}^{+}(1-0)$ lines, indicating the presence of considerable amounts of cold molecular gas. The strongest $\mathrm{H}_{2} \mathrm{O}$ maser in the region (marked with a triangle in Figures 4 to 8; with a $V_{L S R}=-61 \mathrm{~km} \mathrm{~s}^{-1}$ and a peak position at $\alpha(2000)=18^{h} 19^{m} 12^{s} .509 ; \delta(2000)=-20^{\circ} 47^{\prime} 27^{\prime \prime} 42$, Gómez et al. 1995), lies close $\left(2^{\prime \prime}\right)$ to the molecular peak emission of the $\mathrm{C}$ component (see Table 3 ). The LSR velocities of the individual $\mathrm{H}_{2} \mathrm{O}$ maser spots are between -50 to $-72 \mathrm{~km} \mathrm{~s}^{-1}$ (Martí et al. 1999), considerably blueshifted with respect to the $\mathrm{NH}_{3}, \mathrm{HCN}$ and $\mathrm{HCO}^{+}$molecular emission $(+12$ 
$\left.\mathrm{km} \mathrm{s}^{-1}\right)$. A deep $3.5 \mathrm{~cm}$ radio continuum map reveals the presence of a weak $(\sim 0.05 \mathrm{mJy})$ unresolved $\left(\leq 0\right.$ "'2) radio source (VLA 3 in Gómez et al. 1995; $\alpha(2000)=18^{h} 19^{m} 12^{s} .48$; $\left.\delta(2000)=-20^{\circ} 47^{\prime} 27^{\prime \prime} 42\right)$ that is very close to the strongest $\mathrm{H}_{2} \mathrm{O}$ maser. The position of VLA 3 is also located a few arcsec from the position of the $1.4 \mathrm{~mm}$ emission detected by us $\left(\alpha(2000)=18^{h} 19^{m} 12^{s} .13 ; \delta(2000)=-20^{\circ} 47^{\prime} 30^{\prime \prime} 57\right.$; see Fig. 2$)$ and we tentatively propose that they correspond to the same source. Assuming that the radio continuum emission from VLA 3 arises from an HII region, it requires a B3 ZAMS star to maintain its ionization. All the above data support the idea that the $\mathrm{C}$ molecular clump harbors a cluster of stars in an early stage of formation. Mid-IR observations indicate the presence of a pre-main sequence stellar cluster (Aspin \& Geballe 1992; Yamashita et al. 1987) at this position.

The derived parameters of the $\mathrm{C}$ ammonia component, as well as of the other ammonia components, are summarized in Table 4. The $\mathrm{C}$ ammonia source is the warmer component having a rotational temperature of $\sim 24 \mathrm{~K}$. It shows a velocity gradient of $\sim 3 \mathrm{~km} \mathrm{~s}^{-1}$ over an angular scale of $\sim 12^{\prime \prime}$ (see Fig. 9). If due to rotation, it implies a mass of $\sim 30 \mathrm{M}_{\odot}$, which is in good agreement with the mass of $\sim 40 \mathrm{M}_{\odot}$ estimated from the virial assumption (Table 4).

\subsection{The NE component}

The emission from the NE ammonia clump is seen in the velocity range from 10.0 to $10.6 \mathrm{~km} \mathrm{~s}^{-1}$. Neither HCN nor radio continuum emission was detected toward this clump. This component is associated with methanol masers (coincident within the positional error of the masers of $10^{\prime \prime}$; see Figure 3) as well as with weak $\mathrm{HCO}^{+}$emission. The $95 \mathrm{GHz}$ class I methanol masers found are in a velocity range from 12.4 to $13.7 \mathrm{~km} \mathrm{~s}^{-1}$ (Val'tts et al. 2000), slightly redshifted with respect to the LSR velocity of the ammonia clump $\left(\sim 11 \mathrm{~km} \mathrm{~s}^{-1}\right)$. Class I methanol masers are characterized by a lack of radio continuum emission, and may be related with the first stages of massive stellar evolution (Garay \& Lizano 1999). The relatively warm rotational temperature of this component ( $22 \mathrm{~K}$; see Table 4 ) also favors the presence of embedded stars. The molecular mass estimated from the ammonia data is $\sim 1 \mathrm{M}_{\odot}$. On the other hand, the virial mass is aproximately $13 \mathrm{M}_{\odot}$, suggesting that this molecular clump may not be in equilibrium. Most probably, this warm clump is a substructure of the $\mathrm{C}$ component. In fact, from the channel maps of the $(1,1)$ ammonia

emission (Fig. 5) we note that at $11.9 \mathrm{~km} \mathrm{~s}^{-1}$ there are several weak extended structures around the $\mathrm{C}$ component. In this channel map we also observe an $\mathrm{EW}$ filament resembling the "ammonia fingers" in OMC-1 (Wiseman \& Ho 1998). 


\subsection{The $\mathrm{S}$ component}

This molecular clump was detected only in the $(1,1)$ main transition of $\mathrm{NH}_{3}$ and appears located toward the south of the exciting source of the jet. We did not detect emission of $\mathrm{HCO}^{+}$and $\mathrm{HCO}$ at a $3 \sigma$ level of $1.5 \mathrm{Jy}_{\text {beam }}{ }^{-1}$. The ammonia $(1,1)$ line has $\mathrm{V}_{L S R} \sim$ $13 \mathrm{~km} \mathrm{~s}^{-1}$, slightly redshifted with respect to the $\mathrm{C}$ component. The low rotational temperature of this component (10 K; see Table 4) suggests that it does not have embedded an object of large luminosity. The estimates of the molecular mass and the virial mass are very similar, $\sim 13 \mathrm{M}_{\odot}$, suggesting that this clump is probably in equilibrium. It will then be interesting to study this component in detail since it may be a precursor to the formation of stars. Martí et al. (1993) found that along the north-south direction of the thermal jet there are several knots that emit at radio frequencies and in particular the $\mathrm{S}$ ammonia component is close to one of those knots (source VLA 13 from Martí et al. 1993): 21" or 0.18 pc in projection. No counterpart was found at other frequencies for this new $\mathrm{S}$ ammonia clump using the SIMBAD database. An inspection of the 2MASS All Sky Survey shows that there are many near-IR sources in the GGD 27 region. Yet, there is a region of roughly 40" of diameter around the $\mathrm{S}$ ammonia clump where there are no near-IR sources. This suggests that the S ammonia clump is part of a very dark molecular cloud, where apparently there is no star formation going on. The presence of a possible shock excited radio source nearby (source VLA 13) suggests that this clump may belong to the class of irradiated clumps, that is, clumps that are exposed to the strong radiation of a Herbig-Haro object (e.g. Girart et al. 1994, 2002). In these clumps the ammonia emission is strongly enhanced (Viti \& Williams 1999; Viti et al. 2003). If the S clump is an irradiated clump, the estimated mass would be much lower than $13 \mathrm{M}_{\odot}$.

\subsection{Emission Toward the Jet}

Our results show the presence of SO molecular emission and continuum emission at $\mathrm{mm}$ wavelengths in the direction of the exciting source of the $\mathrm{HH} 80-81$ jet. The $5_{5}-4_{4}$ transition of $\mathrm{SO}$ is a tracer of warm $(\geq 40 \mathrm{~K})$ and dense $\left(\sim 10^{6} \mathrm{~cm}^{-3}\right)$ gas (Chernin et al. 1994). The SO emission is detected at $>4 \sigma$ level in both the spectrum and the integrated map of Figure 8. From the SO line emission and assuming optically thin conditions, we obtain a beam-averaged column density of $\mathrm{N}(\mathrm{SO})$ in the range $2.5-3.4 \times 10^{14} \mathrm{~cm}^{-2}$ for a temperature range of 50 to $100 \mathrm{~K}$. Assuming that the $\mathrm{SO} / \mathrm{H}_{2}$ ratio is in the $0.5-2.0 \times 10^{-8}$ range (Helmich \& van Dishoeck 1997), we obtain a beam-averaged $\mathrm{H}_{2}$ column density in the range of $1.3-6.8 \times 10^{22} \mathrm{~cm}^{-2}$, that translates in a mass of $0.7-3.5 \mathrm{M}_{\odot}$. On the other hand, assuming that the dust emission is optically thin and that the dust temperature is in the 50 
- $100 \mathrm{~K}$ range, we derive a total mass from the dust emission of $3.7-1.8 \mathrm{M}_{\odot}$ (Beckwith \& Sargent 1991). We conclude that both the SO and the dust data are consistent with the presence of a few solar masses $\left(1-3 \mathrm{M}_{\odot}\right)$ of gas closely associated with the surroundings of the exciting source of the jet.

We found that no strong ammonia emission was detected toward the jet. The position of the exciting source of the thermal jet lies at the edge of the $\mathrm{C}$ ammonia component (see Figure 4). In the image of the $\mathrm{C}$ component (Figure 6) we see a small enhancement of the $(2,2)$ ammonia emission along the direction of the thermal jet, while there is no emission in the $(1,1)$ image. Figure 10 shows the spectra of the $(1,1)$ and $(2,2)$ inversion transitions at the peak position of the thermal jet. We detect a weak and broad $(2,2)$ ammonia line emission which suggests that warm ammonia gas is present in the vicinity of the exciting source of the thermal jet. From a Gaussian profile fit to the $(2,2)$ spectrum we obtain a peak flux of $4.8 \pm 0.7 \mathrm{mJy}_{\mathrm{beam}}{ }^{-1}$, an LSR line center velocity of $11.5 \pm 0.3 \mathrm{~km} \mathrm{~s}^{-1}$ and a line width of $5.4 \pm 0.8 \mathrm{~km} \mathrm{~s}^{-1}$. Using these parameters for the $(2,2)$ line and a $2 \sigma$ upper limit

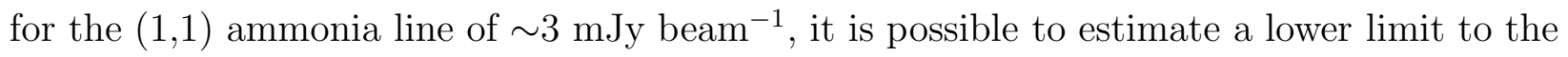
rotational temperature of $\sim 50 \mathrm{~K}$. This is unusually warm molecular gas, that we suggest is being heated by the star that produces the jet. However, the determination of its precise location and nature cannot be addressed with the data presented here. A crude upper limit for the molecular mass associated with the jet can be obtained as follows. From the peak $(2,2)$ line flux density of $4.8 \mathrm{mJy}_{\mathrm{beam}}{ }^{-1}$ and the angular resolution of the observations, we estimate a line brightness temperature of $\sim 0.7 \mathrm{~K}$. Assuming that the excitation temperature is $50 \mathrm{~K}$,this implies $\tau(2,2, m) \simeq 0.014$, an optically thin line. Adopting a line width of 5.4 $\mathrm{km} \mathrm{s}^{-1}$, we derive a total column density of ammonia of $N\left(N H_{3}\right) \simeq 2 \times 10^{14} \mathrm{~cm}^{-2}$. Using the ratio of $\left[H_{2} / \mathrm{NH}_{3}\right]=10^{8}$ used for the ammonia components, this implies $N\left(H_{2}\right) \simeq 2 \times 10^{22}$ $\mathrm{cm}^{-2}$. Finally, assuming that the gas is distributed over a physical size similar to the beam, we obtain a mass estimate of $0.3 \mathrm{M}_{\odot}$. It is unclear if this molecular gas is associated with the jet or with the edge of the $\mathrm{C}$ molecular component. In any case, the mass derived from ammonia for the surroundings of the jet is an order of magnitude smaller than that derived from SO or dust, suggesting that ammonia is strongly depleted.

\section{Conclusions}

We have made high angular resolution VLA and BIMA observations of $\mathrm{NH}_{3}, \mathrm{HCO}^{+}$, $\mathrm{HCN}$, and SO molecular gas as well as 1.4, 3.5 and $7 \mathrm{~mm}$ continuum emission toward the exciting source of HH 80-81. Our main conclusions are summarized as follows.

We detected millimeter continuum emission $(1.4,3.5$ and $7 \mathrm{~mm})$, as well as $\mathrm{SO} 5_{5}-4_{4}$ 
line emission, associated with the exciting source of the HH 80-81 outflow. These emissions may be arising from a circumstellar disk, but higher angular resolution data are required to study the morphology of the emitting dust and gas. This is the first time that SO $5_{5}-4_{4}$ line emission is detected toward the exciting source of HH 80-81, suggesting that this transition may be a good tracer of molecular gas near massive protostars. Only weak ammonia $(2,2)$ emission was detected toward the exciting source of the jet, indicating a temperature $\sim 50$ K. Unfortunately, the precise location and nature of the warm molecular gas traced by the ammonia cannot be determined with the present data.

Three molecular components have been detected in the surroundings of the exciting source of the $\mathrm{HH}$ 80-81 outflow. The brightest of these condensations (the $\mathrm{C}$ component) was detected in $\mathrm{NH}_{3}, \mathrm{HCO}^{+}, \mathrm{HCN}$ and appears associated with $\mathrm{H}_{2} \mathrm{O}$ maser emission suggesting that this component harbors a cluster of young stars. The NE component was detected in $\mathrm{NH}_{3}$ and $\mathrm{HCO}^{+}$and is associated with class I methanol masers. The $\mathrm{S}$ component was detected only in $\mathrm{NH}_{3}$, and seems to be a massive (about $15 \mathrm{M}_{\odot}$ ) and cold clump without ongoing stellar formation. Alternatively, this component may belong to the class of irradiated clumps, with VLA 13 being the source of the radiation.

YG and LFR acknowledge financial support from DGAPA-UNAM and CONACyT, México. JMG is supported by MCyT grant AYA2002-00205. GG acknowledges support from FONDECYT project 1010531. JM also acknowledges partial support by DGI of the Ministerio de Ciencia y Tecnología (Spain) under grant AYA2001-3092, as well as by Junta de Andalucía (Spain) under PAI group FQM322. This research has made use of the SIMBAD database, operated at CDS, Strasbourg, France. The 2MASS project is a collaboration between The University of Massachusetts and the infrared processing Analysis Center (JPL/Caltech), with funding provided primarily by NASA and NSF. 
Table 1. PARAMETERS OF THE BIMA OBSERVATIONS

\begin{tabular}{|c|c|c|c|c|c|c|c|}
\hline \multirow[b]{2}{*}{ Epoch } & \multirow[b]{2}{*}{ Observation } & \multirow{2}{*}{$\begin{array}{c}\nu \\
(\mathrm{GHz})\end{array}$} & \multicolumn{2}{|c|}{ Synthesized Beam } & \multirow{2}{*}{$\begin{array}{c}\text { Bandwidth } \\
(\mathrm{MHz})\end{array}$} & \multirow{2}{*}{$\begin{array}{c}\Delta v^{\mathrm{a}} \\
\left(\mathrm{km} \mathrm{s}^{-1}\right)\end{array}$} & \multirow{2}{*}{$\begin{array}{c}R M S^{\mathrm{b}} \\
\left(\mathrm{Jy} \mathrm{Beam}^{-1}\right)\end{array}$} \\
\hline & & & HPBW & P.A. & & & \\
\hline \multirow[t]{3}{*}{1999 Sep 27} & Continuum & 85.12 & $19^{\prime \prime} 0 \times 6^{\prime \prime} .3$ & $-14^{\circ}$ & 100 & & 0.021 \\
\hline & $\mathrm{HCO}^{+} 1-0$ & 89.18852 & $18^{\prime \prime} \cdot 7 \times 6^{\prime \prime} .1$ & $-14^{\circ}$ & 25 & 0.66 & 0.56 \\
\hline & HCN 1-0 & 88.63185 & $18^{\prime \prime} .3 \times 6{ }^{\prime \prime} .1$ & $-14^{\circ}$ & 25 & 0.66 & 0.52 \\
\hline \multirow[t]{3}{*}{2001 May 6} & Continuum $^{\mathrm{c}}$ & 216.77 & $7^{\prime \prime} .4 \times 22^{\prime \prime} .9$ & $-7^{\circ}$ & 1200 & & 0.038 \\
\hline & $\mathrm{SO} 5_{5}-4_{4}$ & 215.22065 & $7^{\prime \prime} .4 \times 3^{\prime \prime} .1$ & $-9^{\circ}$ & 25 & 0.68 & 1.80 \\
\hline & $\mathrm{H}_{2} \mathrm{CO} 3_{0,3}-2_{0,2}$ & 218.22219 & $7^{\prime \prime} .4 \times 33^{\prime \prime} 1$ & $-9^{\circ}$ & 25 & 0.67 & 2.00 \\
\hline
\end{tabular}

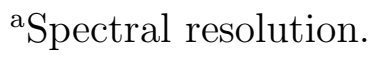

${ }^{b}$ Per channel. The brightness temperature of the $3.4 \mathrm{~mm}$ data is $T_{b}(K) \simeq 1.4 \times 10^{-3} \mathrm{~S}(\mathrm{mJy})$, while for the $1.4 \mathrm{~mm}$ data is $T_{b}(K) \simeq 1.1 \times 10^{-3} S(m J y)$.

'The continuum maps at $1.4 \mathrm{~mm}$ were made using the whole upper and lower sidebands. 
Table 2. DUST EMISSION

\begin{tabular}{crr}
\hline \hline \multirow{2}{*}{$\lambda$} & \multicolumn{2}{c}{ Total Flux (Jy) } \\
\cline { 2 - 3 }$(\mathrm{mm})$ & Thermal Jet & C component \\
\hline 7.0 & $0.014 \pm 0.002$ & $\lesssim 0.006$ \\
3.5 & $0.10 \pm 0.02$ & $\lesssim 0.08$ \\
1.4 & $0.97 \pm 0.11$ & $0.21 \pm 0.05$ \\
\hline
\end{tabular}


Table 3. AMMONIA $(1,1)$ AND $(2,2)$ LINE PARAMETERS

\begin{tabular}{|c|c|c|c|c|c|c|c|}
\hline \multirow{2}{*}{\multicolumn{2}{|c|}{ Component }} & \multicolumn{2}{|l|}{ Peak Position } & \multirow{2}{*}{$\begin{array}{c}\mathrm{V}_{L S R} \\
\left(\mathrm{~km} \mathrm{~s}^{-1}\right)\end{array}$} & \multirow{2}{*}{$\begin{array}{c}\Delta \mathrm{V} \\
\left(\mathrm{km} \mathrm{s}^{-1}\right)\end{array}$} & \multirow{2}{*}{$\begin{array}{l}\text { Flux Density }{ }^{a} \\
\quad(\mathrm{mJy})\end{array}$} & \multirow{2}{*}{$\begin{array}{l}\Theta_{S}^{b} \\
(\prime \prime)\end{array}$} \\
\hline & & $\alpha(2000)$ & $\overline{\delta(2000)}$ & & & & \\
\hline \multirow[t]{3}{*}{ C } & $(1,1 ; \mathrm{m})$ & 181912.54 & -204725.5 & $12.2 \pm 0.1$ & $2.3 \pm 0.1$ & $260 \pm 6$ & 8.9 \\
\hline & $(1,1 ; \mathrm{s})$ & & & $12.1 \pm 0.1$ & $2.6 \pm 0.1$ & $80 \pm 6$ & \\
\hline & $(1,1 ; \mathrm{s})$ & & & $12.2 \pm 0.1$ & $1.9 \pm 0.1$ & $108 \pm 6$ & \\
\hline $\mathrm{C}$ & $(2,2 ; \mathrm{m})$ & 181912.55 & -204725.4 & $12.2 \pm 0.1$ & $2.4 \pm 0.1$ & $188 \pm 6$ & 10.6 \\
\hline \multirow[t]{3}{*}{$\mathrm{NE}$} & $(1,1 ; \mathrm{m})$ & 181913.52 & -204713.2 & $10.6 \pm 0.1$ & $2.1 \pm 0.2$ & $25 \pm 2$ & 3.5 \\
\hline & $(1,1 ; \mathrm{s})$ & & & $11.7 \pm 0.4$ & $3.4 \pm 0.8$ & $8 \pm 2$ & \\
\hline & $(1,1 ; \mathrm{s})$ & & & $11.3 \pm 0.4$ & $3.9 \pm 0.9$ & $8 \pm 2$ & \\
\hline $\mathrm{NE}$ & $(2,2 ; \mathrm{m})$ & 181913.42 & -204713.1 & $10.5 \pm 0.1$ & $1.9 \pm 0.3$ & $14 \pm 2$ & \\
\hline \multirow[t]{3}{*}{ S } & $(1,1 ; \mathrm{m})$ & 181911.94 & -20 4829.3 & $13.0 \pm 0.1$ & $1.6 \pm 0.1$ & $111 \pm 6$ & 6.1 \\
\hline & $(1,1 ; \mathrm{s})$ & & & $12.9 \pm 0.1$ & $1.6 \pm 0.2$ & $67 \pm 6$ & \\
\hline & $(1,1 ; \mathrm{s})$ & & & $13.0 \pm 0.1$ & $1.7 \pm 0.2$ & $58 \pm 6$ & \\
\hline S & $(2,2 ; \mathrm{m})$ & & & & & $<18$ & \\
\hline
\end{tabular}

${ }^{a}$ At the peak of the line.

${ }^{\mathrm{b}}$ Angular size estimated from a Gaussian fit. 
Table 4. DERIVED PARAMETERS OF THE AMMONIA STRUCTURES

\begin{tabular}{lcccccccc}
\hline \hline Component & $\tau_{m}(1,1)$ & $\tau_{m}(2,2)$ & $\begin{array}{c}l^{a} \\
(\mathrm{pc})\end{array}$ & $\begin{array}{c}\mathrm{T}_{\text {rot }} \\
(\mathrm{K})\end{array}$ & $\begin{array}{c}\mathrm{N}\left(\mathrm{NH}_{3}\right) \\
\left(\mathrm{cm}^{-2}\right)\end{array}$ & $\begin{array}{c}\mathrm{n}\left(\mathrm{H}_{2}\right)^{b} \\
\left(\mathrm{~cm}^{-3}\right)\end{array}$ & $\begin{array}{c}\mathrm{M}\left(\mathrm{H}_{2}\right) \\
\left(\mathrm{M}_{\odot}\right)\end{array}$ & $\begin{array}{c}\mathrm{M}_{\text {vir }} \\
\left(\mathrm{M}_{\odot}\right)\end{array}$ \\
\hline $\mathrm{C}$ & 0.77 & 0.49 & 0.073 & 24 & $2.5 \times 10^{15}$ & $1 \times 10^{6}$ & 16 & 40 \\
$\mathrm{NE}$ & 0.39 & 0.20 & 0.029 & 21 & $1.0 \times 10^{15}$ & $1 \times 10^{6}$ & 1 & 13 \\
$\mathrm{~S}$ & 2.63 & $<0.16$ & 0.050 & $<10$ & $4.9 \times 10^{15}$ & $3 \times 10^{6}$ & 15 & 13 \\
\hline
\end{tabular}

${ }^{a}$ Diameter estimated assuming a distance of $1.7 \mathrm{kpc}$.

${ }^{\mathrm{b}}$ Derived assuming $\left[\mathrm{H}_{2} / \mathrm{NH}_{3}\right]=10^{8}$ 


\section{REFERENCES}

Aspin, C., \& Geballe, T. R. 1992, A\&A, 266, 219

Beckwith, S. V. W., \& Sargent, A. I. 1991, ApJ, 381, 250

Beckwith, S. V. W., \& Sargent, A. I. 1996, Nature, 383, 139

Bonnell, I. A., \& Bate, M. 2002, MNRAS, 336, 659

Cesaroni, R., Felli, M., Testi, L., Walmsley, C. M., \& Olmi, L. 1997, A\&A, 325, 725

Chernin, L. M., Masson, C. R., \& Fuller, G. A. 1994, ApJ, 436, 741

Devine, J. D., Bally, J., Reipurth, B., Shepherd, D., \& Watson, A. 1999, AJ, 117, 2919

Garay, G., \& Lizano, S. 1999, PASP, 111, 1049

Garay, G., Brooks, K., Mardones, D., \& Norris, R. P. 2003, ApJ, 587, 739

Girart, J. M., et al. 1994, ApJ, 435, L145.

Girart, J. M., Estalella, R., Viti, S., Williams, D. A., \& Ho, P. T. P. 2001, ApJ, 562, L91

Girart, J. M., Viti, S., Williams, D. A., Estalella, R., \& Ho, P. T. P. 2002, A\&A, 388, 1004

Gómez, Y., Rodríguez, L. F., \& Martí, J. 1995, ApJ, 453, 268

Gyulbudaghian, A. L., Glushkov, Y. L., \& Denisyuk, E. K. 1978, ApJ, 224, L137

Heathcote, S., Reipurth, B., \& Raga, A. C. 1998, AJ, 116, 1940

Helmich, F. P., \& van Dishoeck, E. F. 1997, A\&AS, 124, 205

Kurtz, S. 2000, RMxAAC, 9, 169

Martí, J., Rodríguez, L. F., \& Reipurth, B. 1993, ApJ, 416, 208

Martí, J., Rodríguez, L. F., \& Reipurth, B. 1995, ApJ, 449, 184

Martí, J., Rodríguez, L. F., \& Reipurth, B. 1998, ApJ, 502, 337

Martí, J., Rodríguez, L. F., \& Torrelles, J. M. 1999, A\&A, 345, L5

Reipurth, B., \& Graham, J. A. 1988, A\&A, 202, 219 
Rodríguez, L. F., Moran, J. M., Dickinson, D. F., \& Gyulbudaghian, A. L. 1978, ApJ, 226, 115

Rodríguez, L. F., Moran, J. M., Ho, P. T. P., \& Gottlieb, W. 1980, ApJ, 235, 845

Rodríguez, L. F., Martí, J., Cantó, J., Moran, J. M., \& Curiel, S. 1993, RexMexA\&A, 25, 23

Rodríguez, L. F., Garay, G., Curiel, S., Ramírez, S., Torrelles, J. M., Gómez, Y., \& Velázquez, A. 1994, ApJ, 430, L65

Rodríguez, L. F., et al. 1998, Nature, 395, 355

Rodríguez, L. F., Anglada, G., \& Curiel, S. 1999, ApJS, 125427

Scoville, N. Z., \& Kwan, J. 1976, ApJ, 206, 718

Shepherd, D. S., Watson, A. M., Sargent, A. I., \& Churchwell, E. 1998, ApJ, 507, 861

Shepherd, D. S., Claussen, M. J., \& Kurtz, S. E. 2001, Science, 292, 151

Torrelles, J. M., Ho, P. T. P., Moran, J. M., Rodríguez, L. F., \& Cantó, J. 1986, ApJ, 307, 787

Torrelles, J. M., Gómez, J. F., Rodríguez, L. F., Curiel, S., Ho, P. T. P., \& Garay, G. 1996, ApJ, 457, L107

Trinidad, M. A., et al. 2003, ApJ, 589, 386

Val'tss, I. E., Ellingsen, S. P., Slysh, V. I., Kalenskii, S. V., Otrupcek, R., \& Larionov, G. M. 2000, MNRAS, 317, 315

Viti, S., \& Williams, D. A. 1999, MNRAS, 310, 517

Viti, S., Girart, J. M., Garrod, R., Williams, D. A., \& Estalella, R. 2003, A\&A, 399, 187

Wilner, D. J., Ho, P. T. P., \& Rodríguez, L. F. 1996, ApJ470, L117

Wilner, D. J., Reid, M. J., \& Menten, K. M. 1999, ApJ, 513, 775

Wilner, D. J., Ho, P. T. P., Kastner, J. H., \& Rodríguez, L. F. 2000, ApJ, 534, L101

Wiseman, J. J., \& Ho, P.T.P. 1998, ApJ, 502, 676

Yamashita, T., Suzuki, H., Kaifu, N., Tamura, M., Mountain, C. M., \& Moore, T. J. T. 1989, ApJ, 347, 894 
Zhang, Q., Hunter, T. R., \& Sridharan, T. K. 1998, ApJ, 505, L151

Zhang, Q., Hunter, T. R., Sridharan, T. K., \& Ho, P. T. P. 2002, ApJ, 566, 982 


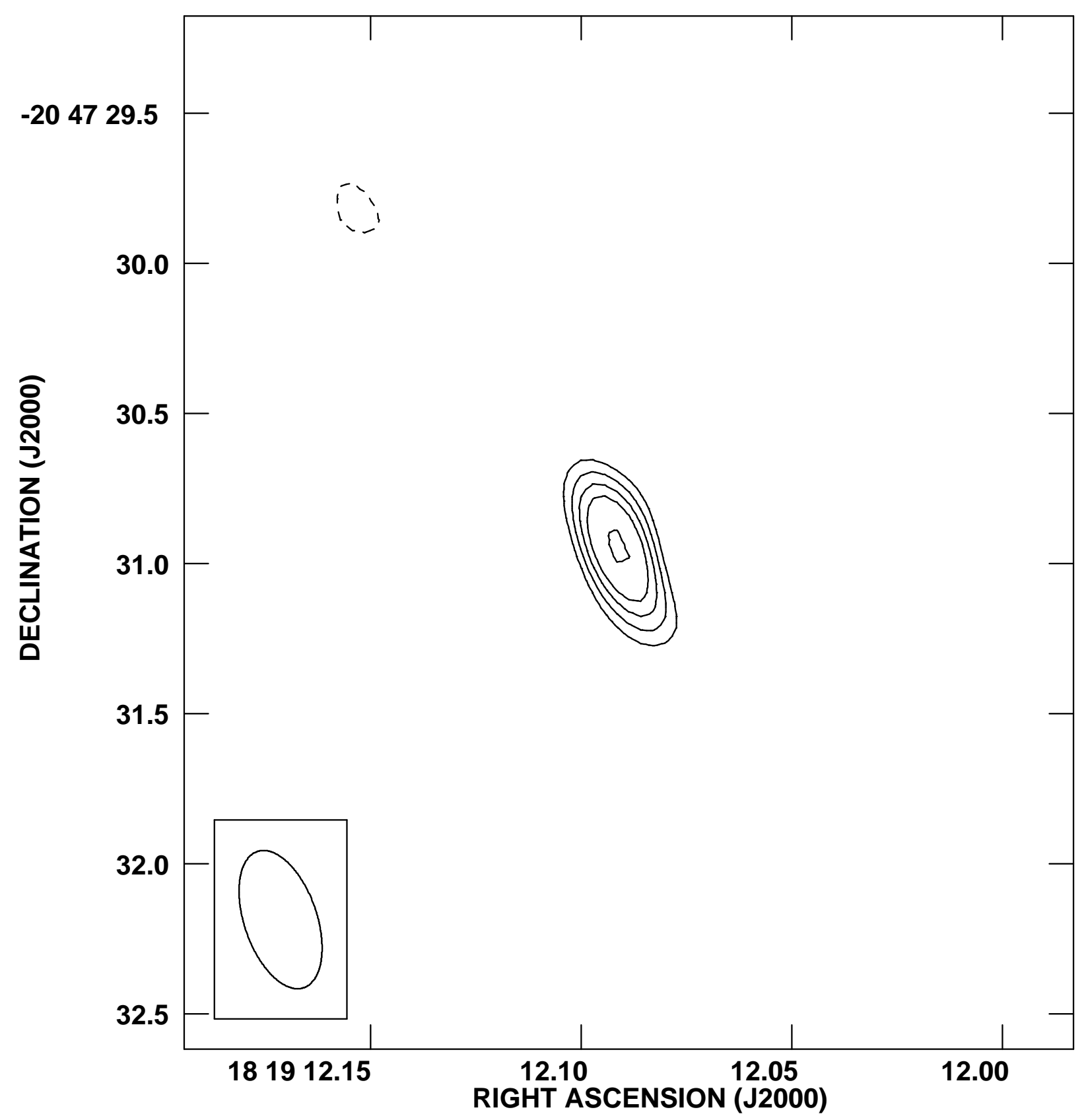

Fig. 1.- VLA image made at $7 \mathrm{~mm}$ of the exciting source of the HH 80-81 outflow. Contours are $-3,3,4,5,6$, and $8 \times 1.3 \mathrm{mJy}_{\text {beam }}^{-1}$, the $\mathrm{rms}$ noise of the image. 


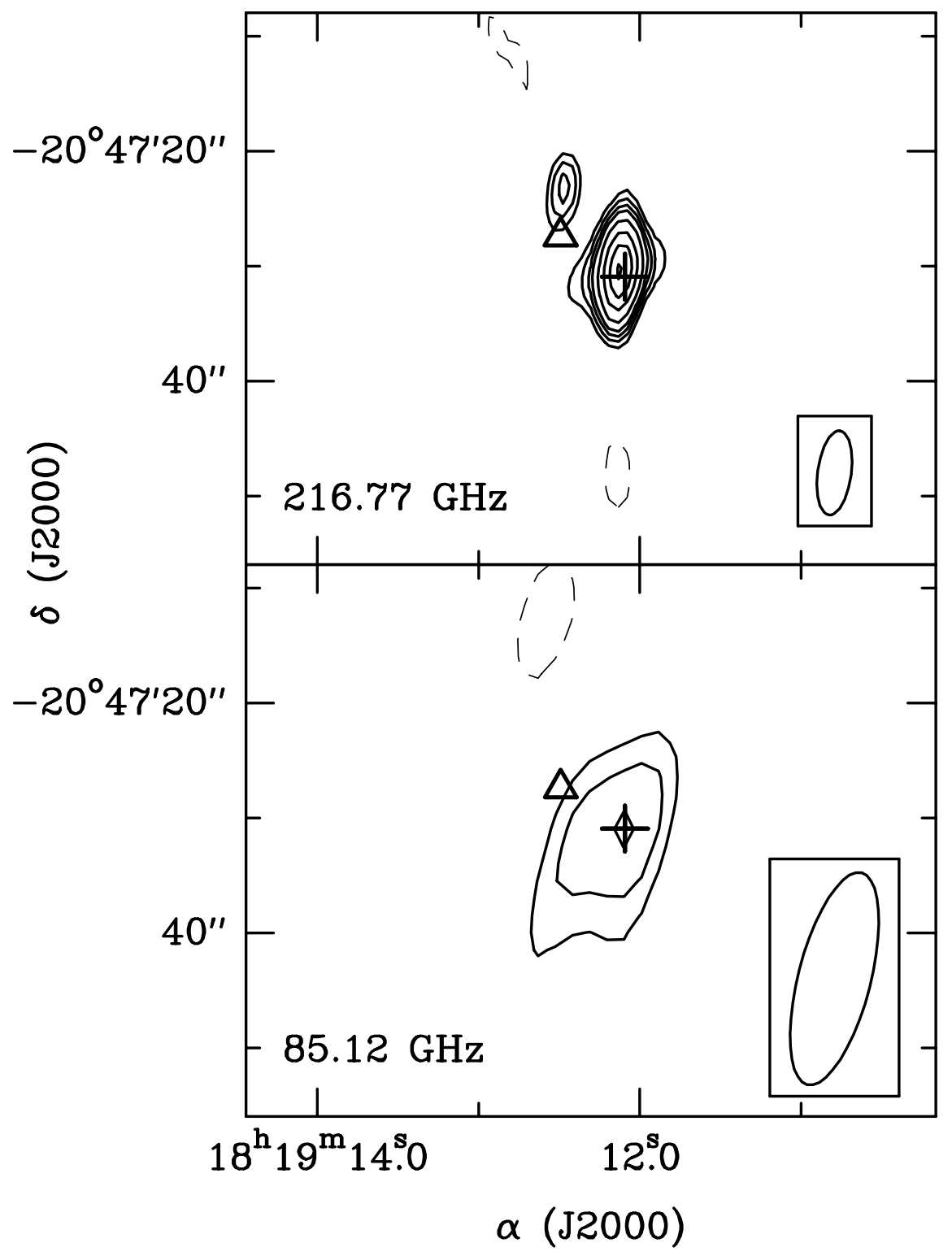

Fig. 2.- Images of the BIMA continuum emission at $1.4 \mathrm{~mm}$ (top panel) and $3.5 \mathrm{~mm}$ (bottom panel). The contour levels are $-3,3,4,5,68,11,14$ and $17 \times 0.038$ and $0.021 \mathrm{Jy}_{\mathrm{beam}^{-1}}$, the $\mathrm{rms}$ noise of the $1.4 \mathrm{~mm}$ and $3.5 \mathrm{~mm}$ maps, respectively. The cross indicates the position of the thermal jet and the triangle marks the position of the $\mathrm{H}_{2} \mathrm{O}$ maser. The synthesized beams are shown in the bottom right corner of each panel. 


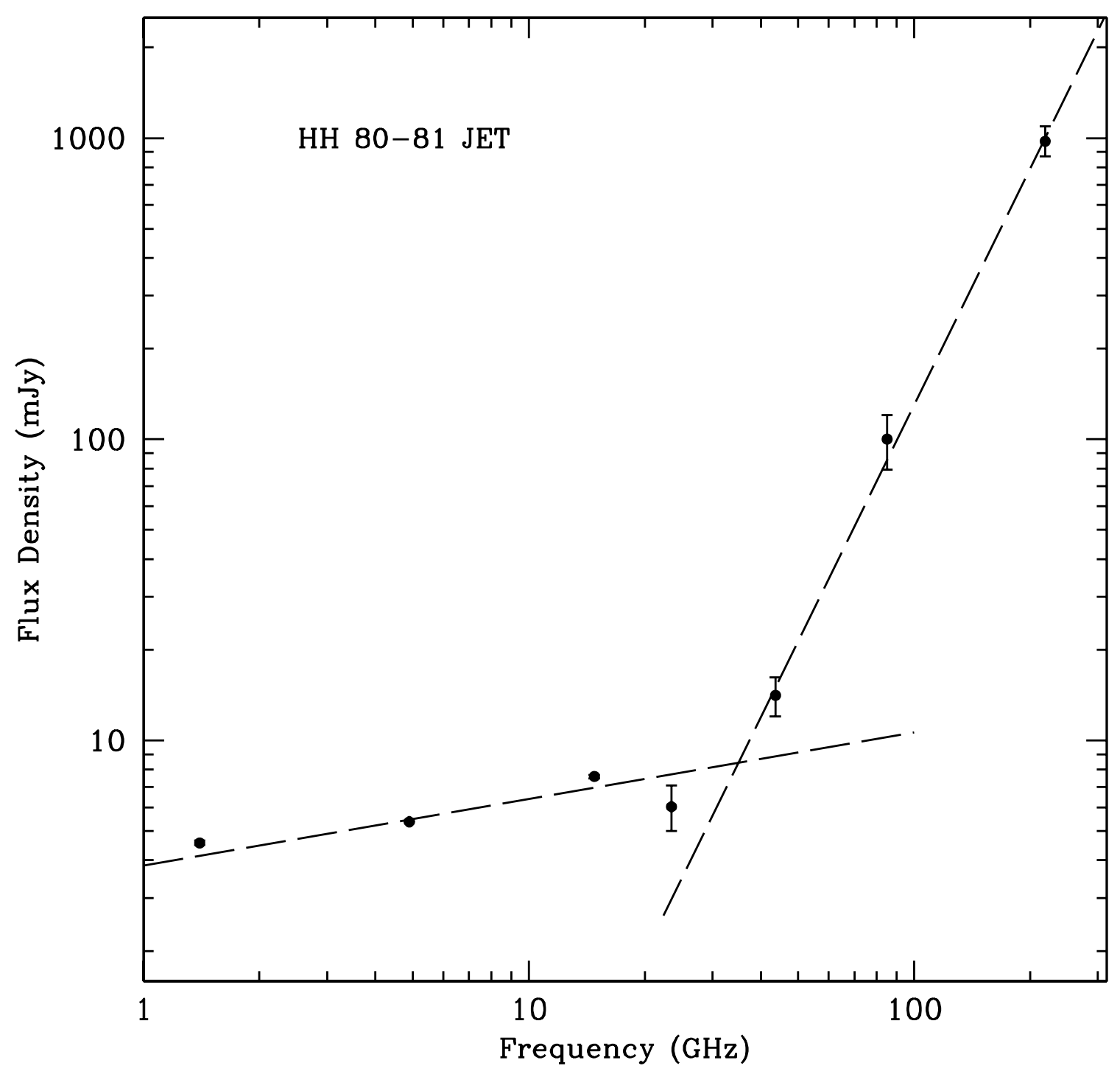

Fig. 3.- Continuum $\mathrm{cm}$ and $\mathrm{mm}$ spectrum of the region associated with the exciting source. The dashed line to the left is a linear fit to the four lower frequency data points, while the dashed line to the right is a linear fit to the three higher frequencies data points. 


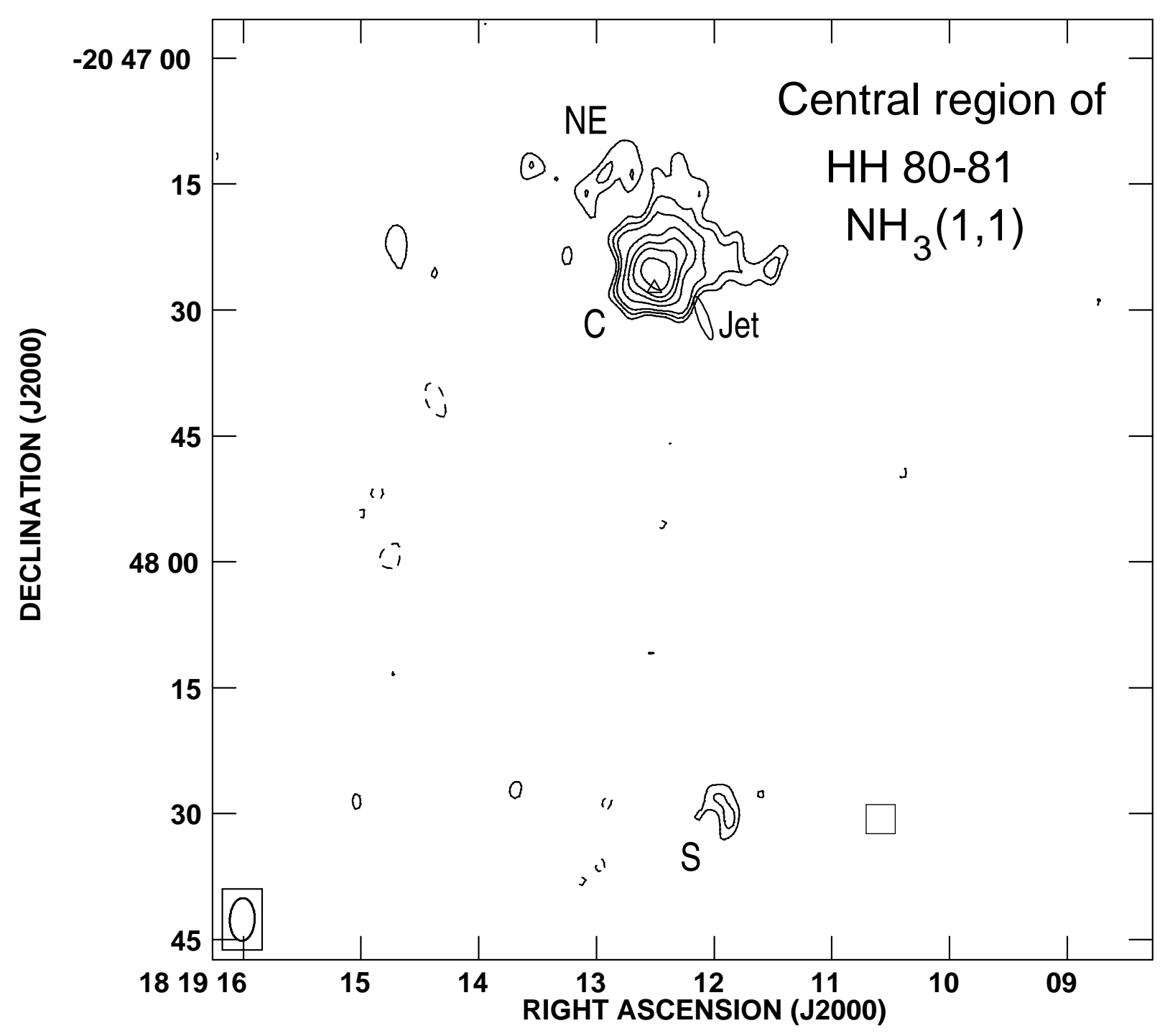

Fig. 4.- Image of the $\mathrm{NH}_{3}(1,1)$ velocity-integrated (from 10.0 to $14.3 \mathrm{~km} \mathrm{~s}^{-1}$ ) line emission from the surroundings of the exciting source of the HH 80-81 region. Contour levels are -3 , $3,4,5,7,9,11$ and $14 \times 1.6 \mathrm{mJy}_{\text {beam }}^{-1}$, the $\mathrm{rms}$ noise of the image. This image is not corrected for the response of the primary beam and the $\mathrm{S}$ component appears weaker than it really is. The parameters given in Table 3 are corrected for the primary beam response. The ellipse indicates the position and alignment of the thermal jet, the triangle marks the $\mathrm{H}_{2} \mathrm{O}$ maser position taken from Gómez et al. (1995) and the small square to the south indicates the position of the radio continuum source VLA 13 corresponding to a knot of the jet (Martí et al. 1993). 

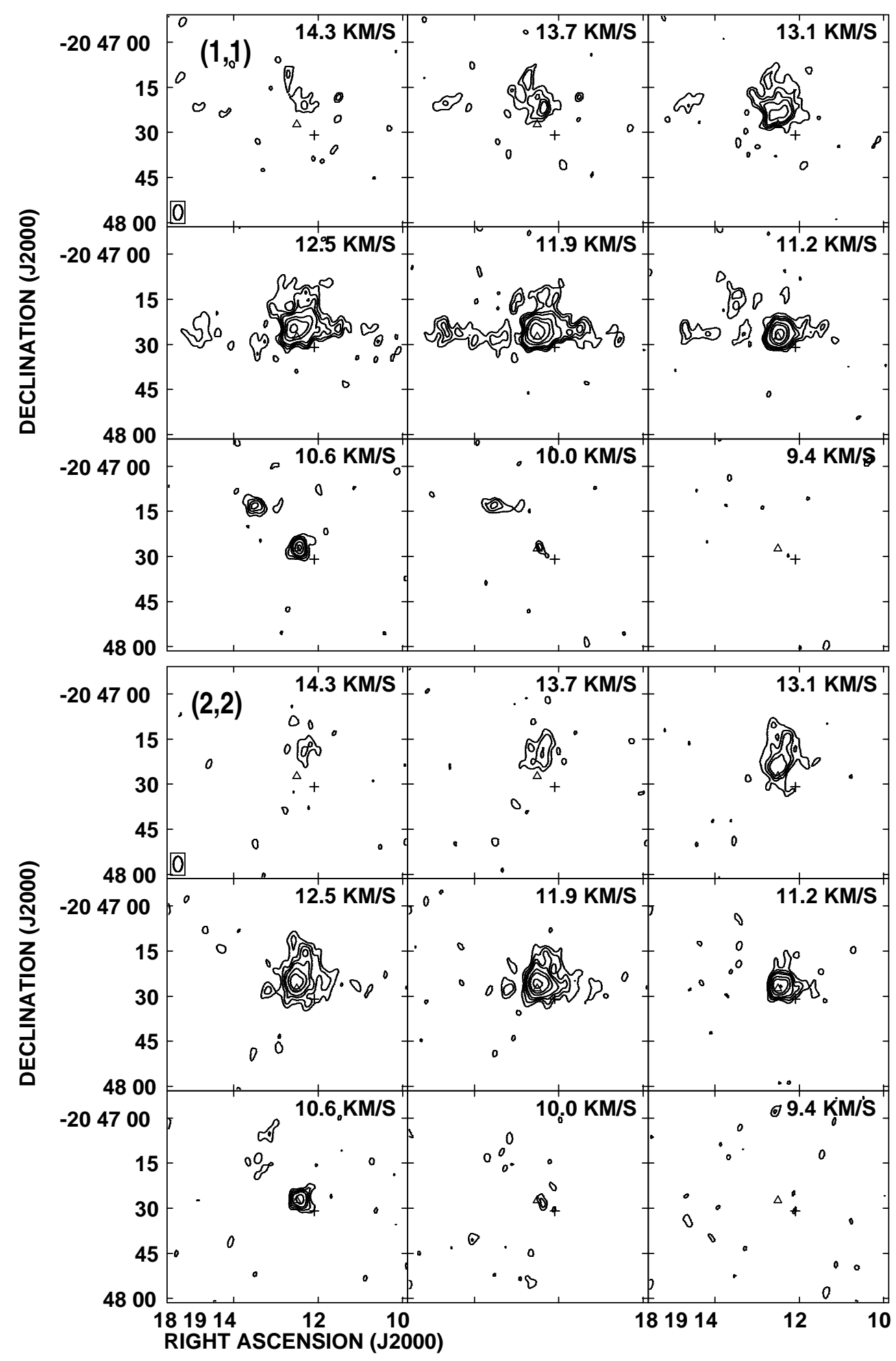

Fig. 5.- Channel maps of the $(1,1)$ (top) and $(2,2)$ (bottom) inversion transition lines of ammonia toward the $\mathrm{HH}$ 80-81 region. Contour levels are 4, 6, 8, 10, 15, 20, 30 and $50 \times$ 1.5 and $1.4 \mathrm{mJy}_{\text {beam }}{ }^{-1}$, respectively. The angular resolution is $5 . \prime 1 \times 33^{\prime \prime} 0$ at P.A. $=-2^{\circ}$. The small cross indicates the position of the thermal jet and the triangle marks the position of the $\mathrm{H}_{2} \mathrm{O}$ maser. 

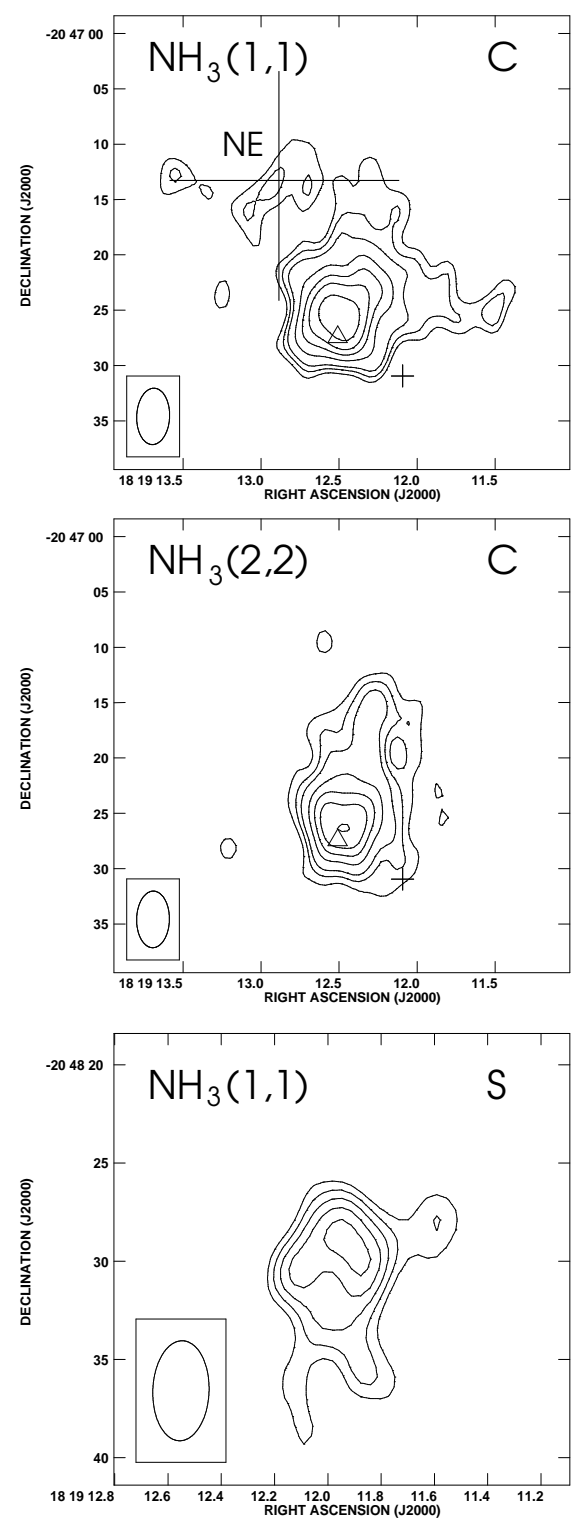

Fig. 6.- Velocity integrated images of the new ammonia components; C, NE and S components. (Top and middle) $\mathrm{C}$ and NE clumps in the $(1,1)$ and $(2,2)$ ammonia emission integrated in a velocity range from 10.0 to $14.3 \mathrm{~km} \mathrm{~s}^{-1}$. The contour levels are $-3,3,4$,

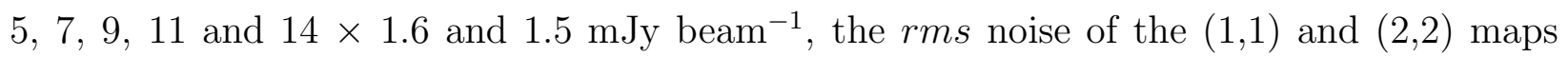
respectively. The small cross indicates the position of the thermal jet, the triangle marks the position of the $\mathrm{H}_{2} \mathrm{O}$ masers and the large cross indicates the position and error of the methanol masers taken from Val'tts et al. (2000). (Bottom) Ammonia (1,1) emission of the $\mathrm{S}$ component integrated in a velocity range from 11.9 to $13.7 \mathrm{~km} \mathrm{~s}^{-1}$. The contour levels

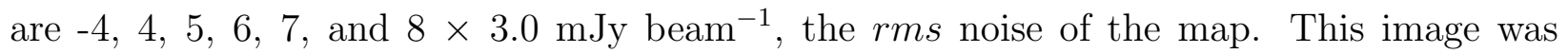
corrected for the response of the primary beam. The $\mathrm{S}$ component was detected only in the $(1,1)$ ammonia transition. 


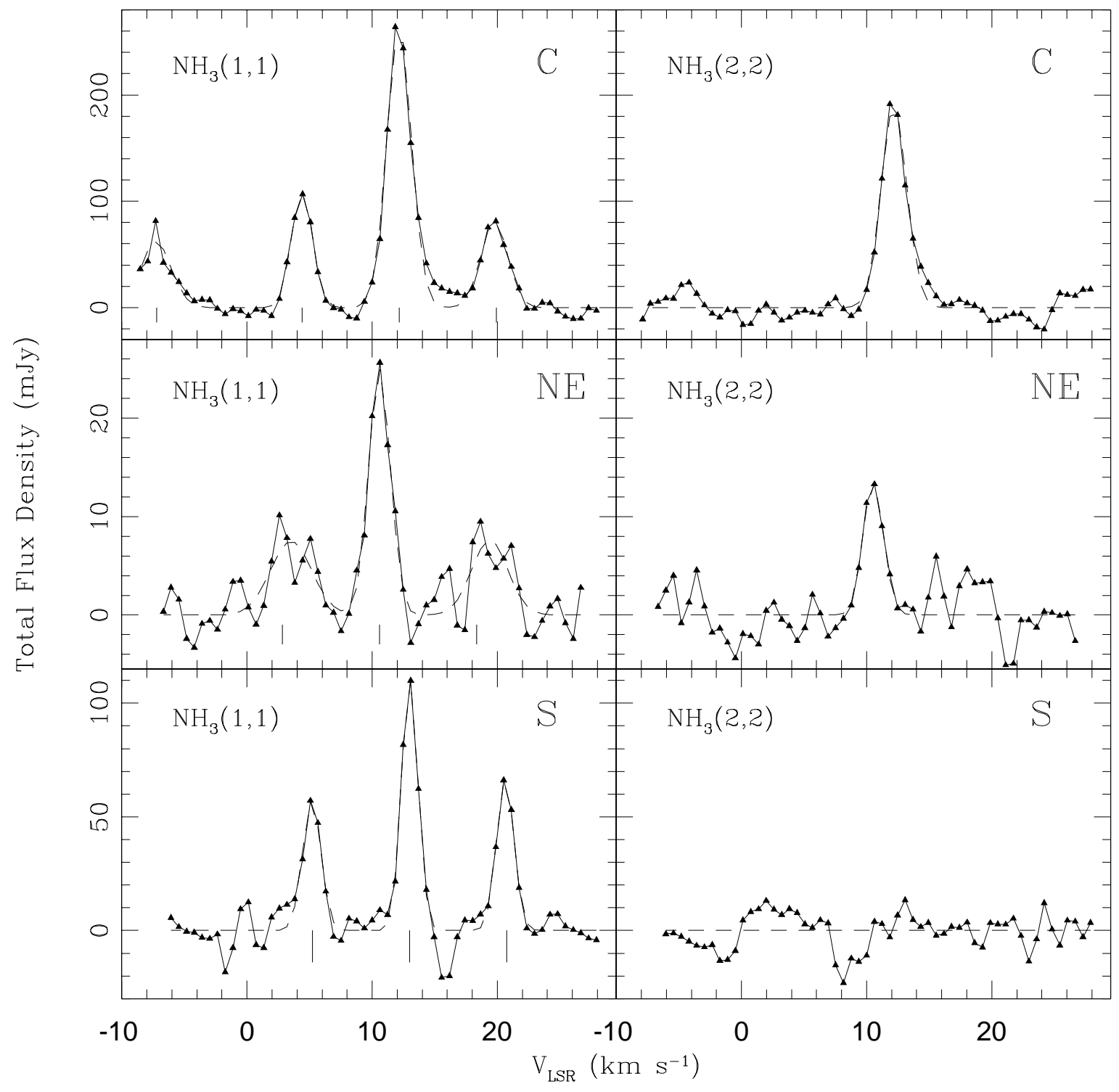

Fig. 7.- Integrated spectra in the $(1,1)$ and $(2,2)$ lines of $\mathrm{NH}_{3}$ from the different molecular components in the HH 80-81 region. The small vertical bars indicate the expected velocities of the main and satellite $(1,1)$ ammonia lines. 

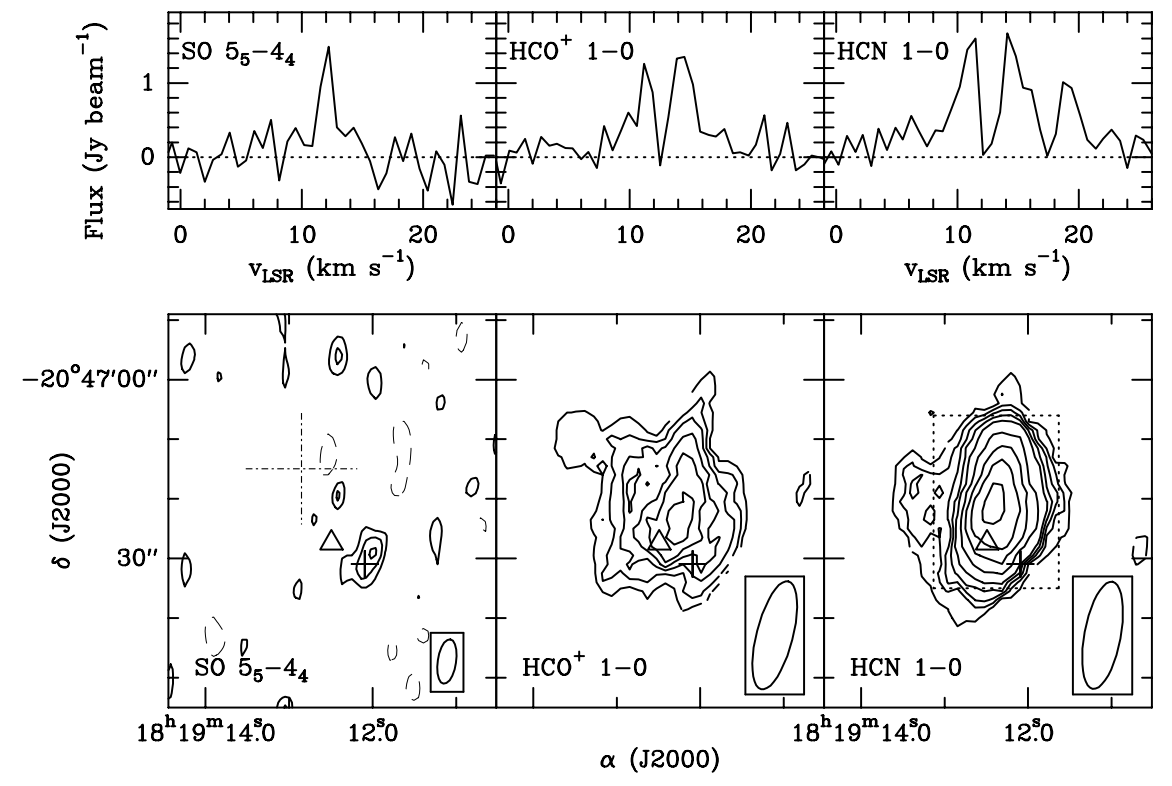

Fig. 8.- Bottom panels. Images of the BIMA integrated emission of the $\mathrm{SO} 5_{5}-4_{4}$ (left panel), $\mathrm{HCO}^{+} 1-0$ (central panel), $\mathrm{HCN} 1-0$ (right panel) lines. The SO map shows the integrated emission in a velocity range from 10.0 to $14.3 \mathrm{~km} \mathrm{~s}^{-1}$. The $\mathrm{HCO}^{+}$and $\mathrm{HCN}$ maps show the integrated emission in a velocity range from 9.5 to $15.7 \mathrm{~km} \mathrm{~s}^{-1}$. The contour levels are $-2,2,3,4,5,6,8,10,12$ and $14 \times 3.2,0.15,0.13 \mathrm{Jy}_{\text {beam }}{ }^{-1}$, the $\mathrm{rms}$ noise of the $\mathrm{SO}, \mathrm{HCO}^{+}$and $\mathrm{HCN}$ maps, respectively. The small cross indicates the position of the exciting source of the thermal jet and the triangle marks the position of the $\mathrm{H}_{2} \mathrm{O}$ maser. The large cross indicates the position and error of the methanol masers taken from Val'tts et al. (2000). The synthesized beams are shown in the bottom right corner of each panel. Top panels. Spectra of the SO $55^{-4} 4_{4}$ (left panel), $\mathrm{HCO}^{+} 1-0$ (central panel), HCN 1-0 (right panel) lines. The SO spectrum was taken at the position of the thermal radio jet and divided by a factor of 5 in order to put it at the same scale than the $\mathrm{HCO}^{+}$and $\mathrm{HCN}$ spectra that are weaker than the $\mathrm{SO}$. For the $\mathrm{HCO}^{+}$and $\mathrm{HCN}$ the spectra was obtained from the averaged emission within the dashed box shown in the HCN map. 


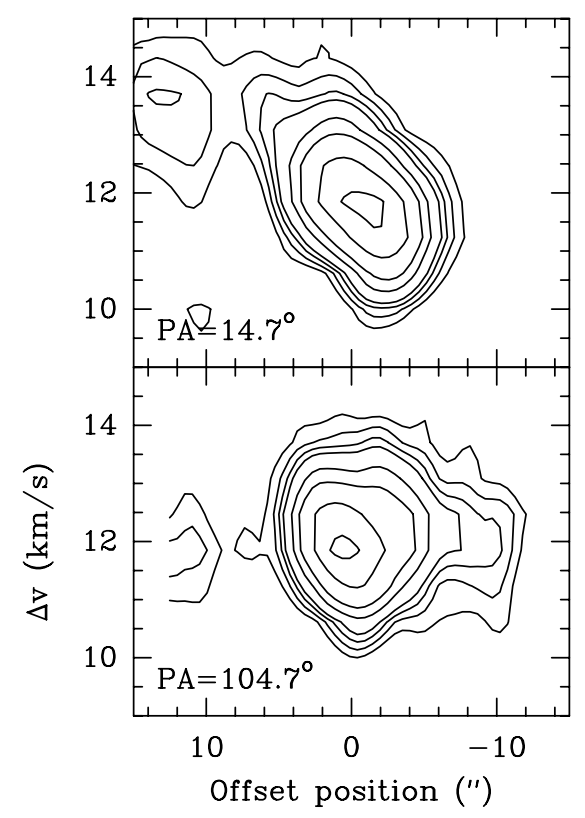

Fig. 9.- Position-velocity diagrams of the $\mathrm{NH}_{3}$ (1,1) emission from the $\mathrm{C}$ component along the major axis direction at P.A. $=14.7^{\circ}$ (top) and along the minor axis direction P.A. $=104.7^{\circ}$ passing through $\alpha(2000)=18^{h} 19^{m} 12.5 ; \delta(2000)=-20^{\circ} 47^{\prime} 24^{\prime \prime} .9$. The contour levels are $-4,4,6,8,10,15,20,30$, and $40 \times 1.4$ mJy beam $^{-1}$. 


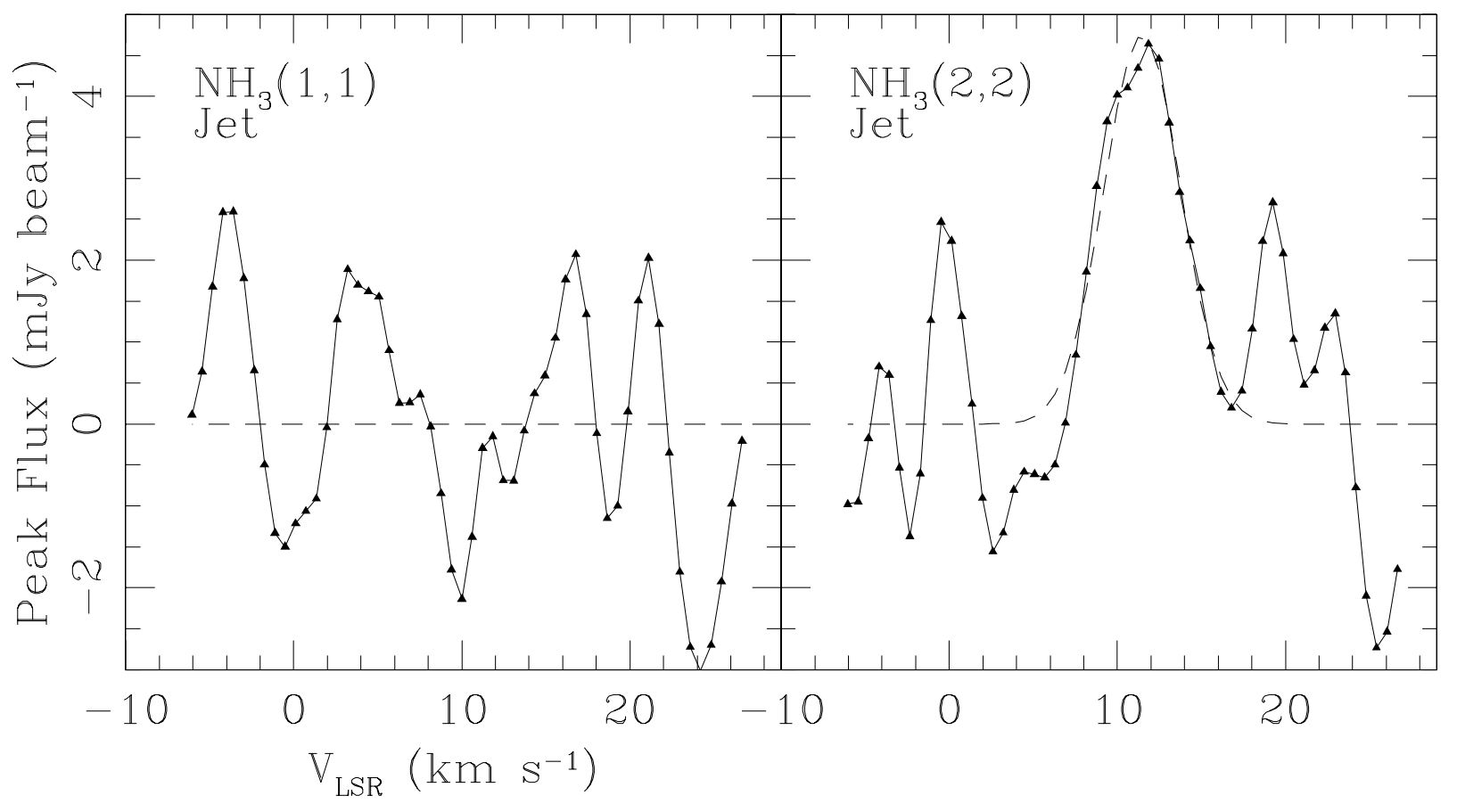

Fig. 10.- Ammonia spectra in the $(1,1)$ and $(2,2)$ lines at the peak position of the HH 80-81 thermal jet: $\alpha(2000)=18^{h} 19^{m} 12^{s} .509 ; \delta(2000)=-20^{\circ} 47^{\prime} 27^{\prime \prime}$. 42 . Only weak and broad $(2,2)$ emission was detected. 